Article

\title{
Iterative Sequences for a Finite Number of Resolvent Operators on Complete Geodesic Spaces
}

\author{
Kengo Kasahara * and Yasunori Kimura \\ Department of Information Science, Toho University, Miyama, Funabashi, Chiba 274-8510, Japan; \\ yasunori@is.sci.toho-u.ac.jp \\ * Correspondence: 7518001k@st.toho-u.jp
}

\begin{abstract}
We consider Halpern's and Mann's types of iterative schemes to find a common minimizer of a finite number of proper lower semicontinuous convex functions defined on a complete geodesic space with curvature bounded above.
\end{abstract}

Keywords: geodesic space; convex minimization problem; resolvent; common fixed point; iterative scheme

\section{Introduction}

We consider finding a common fixed point of a finite number of resolvents operators for proper lower semicontinuous convex functions on a geodesic space. To find this point, we often use iterative schemes. We focus on Mann's [1] and Halpern's [2] iterative schemes. We know many authors have considered these schemes by using nonexpansive mappings. In a Banach space, Reich [3] proved weak convergence of Mann-type iteration, and Takahashi and Tamura [4] proved that by using two nonexpansive mappings. In a Hilbert space, Wittmann [5] proved strong convergence of the Halpern-type iteration.

We also know many researchers have proved iterative schemes on geodesic spaces. In a CAT(0) space, Dhompongsa and Panyanak [6] proved $\Delta$-convergence of Mann's iterative scheme, and Saejung [7] also proved convergence of Halpern's iterative scheme. We know a large number of results by using Mann's and Halpern's iterative schemes in a CAT(1) space. Piątek [8] considered Halpern's iterative scheme by using a nonexpansive mapping in CAT(1) space. Kimura and Satô [9] proved that by using a strongly quasi-nonexpansive and $\Delta$-demiclosed mapping in a complete CAT(1) space. Kimura, Saejung, and Yotkaew [10] also proved convergence of Halpern's iterative schemes under the same setting. Kimura and Kohsaka [11] proved convergence of Mann and Halpern types of iterative schemes with a sequence of resolvent operators for a single proper lower semicontinuous convex function. We are particularly interested in these results [9-11], and obtain Theorems 1 and 2 with a finite number of resolvent operators in a complete CAT(1) space.

In a Hilbert space, the resolvent operator $J_{f}$ is defined as follows. Let $f$ be a proper tral with regard to jurisdictional clai$\mathrm{ms}$ in published maps and institutional affiliations.

Copyright: $\odot 2021$ by the authors. Licensee MDPI, Basel, Switzerland. This article is an open access article distributed under the terms and conditions of the Creative Commons Attribution (CC BY) license (https:// creativecommons.org/licenses/by/ $4.0 /)$. lower semicontinuous convex function from a Hilbert space $H$ to $]-\infty,+\infty]$. Then, $J_{f}$ is defined by

$$
J_{f} x=\underset{y \in H}{\operatorname{argmin}}\left\{f(y)+\frac{1}{2}\|y-x\|^{2}\right\}
$$

for all $x \in H$. We know the resolvent $J_{f}$ is a single-valued mapping from $H$ to $H$ and it is nonexpansive. For a proper lower semicontinuous convex function $f$ from a complete CAT(0) space $X$ into ] $-\infty,+\infty$ ], Jost [12] and Mayer [13] defined the resolvent $R_{f}$ of $f$ by

$$
R_{f} x=\underset{y \in X}{\operatorname{argmin}}\left\{f(y)+\frac{1}{2} d(y, x)^{2}\right\}
$$


for all $x \in X$. We also know the resolvent $R_{f}$ is a single-valued mapping from $X$ to $X$ and it is nonexpansive. In this paper, we use the resolvent in a complete CAT(1) space defined by Kimura and Kohsaka [11,14].

\section{Preliminaries}

Let $(X, d)$ be a metric space. For $x, y \in X$, a geodesic between $x$ and $y$ is an isometric mapping $c:[0, d(x, y)] \rightarrow X$ with $c(0)=x$ and $c(d(x, y))=y$. We say $X$ is an $r$-geodesic space for $r>0$ if a geodesic exists for every pair of points in $X$ satisfying $d(x, y)<r$. Further, a metric space $X$ is said to be $r$-uniquely geodesic if such a geodesic is unique for each pair of points satisfying $d(x, y)<r$. The image of a unique geodesic between $x$ and $y$ is denoted by $[x, y]$.

For an $r$-uniquely geodesic space $X$, the convex combination between $x, y \in X$ with $d(x, y)<r$ is naturally defined. That is, for $\alpha \in[0,1]$, we denote by $\alpha x \oplus(1-\alpha) y$ the point $c((1-\alpha) d(x, y))$, where $c$ is a geodesic between $x$ and $y$. It follows that

$$
d(\alpha x \oplus(1-\alpha) y, x)=(1-\alpha) d(x, y) \text { and } d(\alpha x \oplus(1-\alpha) y, y)=\alpha d(x, y) .
$$

A subset $C$ of $X$ is said to be $r$-convex if $\alpha x \oplus(1-\alpha) y \in C$ for every $x, y \in C$ with $d(x, y)<r$ and $\alpha \in[0,1]$.

If $X$ is $r$-geodesic for any $r>0$, then $X$ is simply called a geodesic space. A uniquely geodesic space and a convex subset are also defined in the same way.

Let $X$ be a uniquely geodesic space and $x, y, z \in X$. For a triangle $\triangle(x, y, z)=[y, z] \cup$ $[z, x] \cup[x, y] \subset X$ satisfying $d(y, z)+d(z, x)+d(x, y)<2 \pi$, we define its comparison triangle $\triangle(\bar{x}, \bar{y}, \bar{z})$ in the two-dimensional unit sphere $\mathbb{S}^{2}$ by the triangle such that each corresponding edge has the same length as that of the original triangle. Using this notion, we call $X$ a CAT(1) space if for every $x, y, z \in X, p, q \in \triangle(x, y, z)$, and their corresponding points $\bar{p}, \bar{q} \in \mathbb{S}^{2}$, the following relation is satisfied,

$$
d(p, q) \leq d_{\mathbb{S}^{2}}(\bar{x}, \bar{y})
$$

where $d_{\mathbb{S}^{2}}$ is the spherical metric on $\mathbb{S}^{2}$.

The following results are fundamental and important for our work.

Lemma 1 (Kimura-Satô [15]). Let $X$ be a CAT(1) space. Then, for every $x, y, z \in X$ with $d(x, y)+d(y, z)+d(z, x)<2 \pi$ and $\alpha \in[0,1]$, the following inequality holds,

$$
\cos d(x, w) \sin d(y, z) \geq \cos d(x, y) \sin (\alpha d(y, z))+\cos d(x, z) \sin ((1-\alpha) d(y, z)),
$$

where $w=\alpha y \oplus(1-\alpha) z$.

Lemma 2 (Kimura-Satô [9]). Let $X$ be a CAT(1) space. Then, for every $x, y, z \in X$ with $d(x, y)+d(y, z)+d(z, x)<2 \pi$ and $\alpha \in[0,1]$, the following inequality holds,

$$
\cos d(x, w) \geq \alpha \cos d(x, y)+(1-\alpha) \cos d(x, z),
$$

where $w=\alpha y \oplus(1-\alpha) z$.

Lemma 3 (Kimura-Satô [9]). Let X be a CAT(1) space such that $d\left(v, v^{\prime}\right)<\pi$ for every $v, v^{\prime} \in X$. Let $\alpha \in[0,1]$ and $u, y, z \in X$. Then,

$$
\begin{aligned}
& 1-\cos d(\alpha u \oplus(1-\alpha) y, z) \\
& \quad \leq(1-\beta)(1-\cos d(y, z))+\beta\left(1-\frac{\cos d(u, z)}{\sin d(u, y) \tan \left(\frac{\alpha}{2} d(u, y)\right)+\cos d(u, y)}\right),
\end{aligned}
$$


where

$$
\beta= \begin{cases}1-\frac{\sin ((1-\alpha) d(u, y))}{\sin d(u, y)} & (u \neq y), \\ \alpha & (u=y) .\end{cases}
$$

Let $\left\{x_{n}\right\} \subset X$ be a bounded sequence. We say a point $z \in X$ is an asymptotic center of $\left\{x_{n}\right\}$ if it is a minimizer of the function $\lim \sup _{n \rightarrow \infty} d\left(x_{n}, \cdot\right)$, that is,

$$
\limsup _{n \rightarrow \infty} d\left(x_{n}, z\right) \leq \limsup _{n \rightarrow \infty} d\left(x_{n}, y\right)
$$

for every $y \in X$. If $z \in X$ is the unique asymptotic center of all subsequences of $\left\{x_{n}\right\}$, then we say $\left\{x_{n}\right\}$ is $\Delta$-convergent to a $\Delta$-limit $z$. We know that in a CAT(1) space, every sequence $\left\{x_{n}\right\}$ satisfying $\inf _{y \in X} \lim \sup _{n \rightarrow \infty} d\left(x_{n}, y\right)<\pi / 2$ has a unique asymptotic center and a $\Delta$-convergent subsequence.

Let $X$ be a CAT(1) space and $T: X \rightarrow X$. The set of all fixed points of $T$ is denoted by $F(T)$. Namely, $F(T)=\{z \in X: z=T z\}$. $T$ is said to be quasi-nonexpansive if $F(T) \neq \varnothing$ and $d(T x, z) \leq d(x, z)$ for every $x \in X$ and $z \in F(T)$. A quasi-nonexpansive mapping $T$ is said to be strongly quasi-nonexpansive if $\lim _{n \rightarrow \infty} d\left(x_{n}, T x_{n}\right)=0$ whenever $\left\{x_{n}\right\} \subset X$ satisfies $\sup _{n \in \mathbb{N}} d\left(x_{n}, p\right)<\pi / 2$ and $\lim _{n \rightarrow \infty}\left(\cos d\left(x_{n}, p\right) / \cos d\left(T x_{n}, p\right)\right)=1$ for every $p \in F(T)$.

A mapping $T$ is said to be $\Delta$-demiclosed if $z \in F(T)$ whenever $\left\{x_{n}\right\}$ is $\Delta$-convergent to $z$ and $\lim _{n \rightarrow \infty} d\left(x_{n}, T x_{n}\right)=0$.

Following [16], we define the notions of a strongly quasi-nonexpansive sequence and a $\Delta$-demiclosed sequence on CAT(1) spaces as follows. Let $\left\{T_{n}\right\}$ be a sequence of mappings from $X$ to $X .\left\{T_{n}\right\}$ is said to be a strongly quasi-nonexpansive sequence if each $T_{n}$ is quasi-nonexpansive and $\lim _{n \rightarrow \infty} d\left(x_{n}, T_{n} x_{n}\right)=0$ whenever $\sup _{n \in \mathbb{N}} d\left(x_{n}, p\right)<\pi / 2$ and $\lim _{n \rightarrow \infty}\left(\cos d\left(x_{n}, p\right) / \cos d\left(T_{n} x_{n}, p\right)\right)=1$ for every $p \in \bigcap_{n=1}^{\infty} F\left(T_{n}\right) .\left\{T_{n}\right\}$ is said to be a $\Delta$-demiclosed sequence if $z \in \bigcap_{n=1}^{\infty} F\left(T_{n}\right)$ whenever $\left\{x_{n}\right\}$ is $\Delta$-convergent to $z$ and $\lim _{n \rightarrow \infty} d\left(x_{n}, T_{n} x_{n}\right)=0$.

Let $X$ be a complete CAT(1) space and $C \subset X$ a nonempty closed $\pi$-convex subset such that $d(x, C)=\inf _{y \in C} d(x, y)<\pi / 2$ for every $x \in X$. Then, for each $x \in X$, there exists a unique point $y_{x} \in C$ satisfying $d\left(x, y_{x}\right)=\inf _{y \in C} d(x, y)$. Using this point, we define a metric projection $P_{C}: X \rightarrow C$ by $P_{C} x=y_{x}$ for $x \in X$.

Let $X$ be a complete CAT(1) space such that $d\left(v, v^{\prime}\right)<\pi / 2$ for every $v, v^{\prime} \in X$. Let $f: X \rightarrow]-\infty,+\infty]$ be a proper lower semicontinuous convex function. The resolvent $R_{f}$ of $f$ is defined by

$$
R_{f} x=\underset{y \in X}{\operatorname{argmin}}(f(y)+\tan d(y, x) \sin d(y, x))
$$

for all $x \in X$; (see in [14]). We know that $R_{f}$ is a single-valued mapping from $X$ to $X$. We also know that the resolvent $R_{f}$ is strongly quasi-nonexpansive and $\Delta$-demiclosed such that $F\left(R_{f}\right)=\operatorname{argmin}_{x \in X} f$ (see $\left.[11,14]\right)$.

We recall some lemmas useful for our results.

Lemma 4 (Kimura-Satô [17]). Let $X$ be a complete CAT(1) space such that $d(u, v)<\pi / 2$ for all $u, v \in X$. Let $S, T$ be quasi-nonexpansive mappings from $X$ to $X$ with $F(S) \cap F(T) \neq \varnothing$. Then, for every $\alpha \in] 0,1[, F(S) \cap F(T)=F(\alpha S \oplus(1-\alpha) T)$ and the mapping $\alpha S \oplus(1-\alpha) T$ is quasi-nonexpansive.

Lemma 5 (He-Fang-López-Li [18]). Let $X$ be a complete CAT(1) space and $p \in X$. If a sequence $\left\{x_{n}\right\}$ in $X$ satisfies that $\limsup _{n \rightarrow \infty} d\left(x_{n}, p\right)<\pi / 2$ and that $\left\{x_{n}\right\}$ is $\Delta$-convergent to $x \in X$, then $d(x, p) \leq \liminf _{n \rightarrow \infty} d\left(x_{n}, p\right)$. 
Lemma 6 (Saejung-Yotkaew [19], Aoyama-Kimura-Kohsaka [20]). Let $\left\{s_{n}\right\}$ and $\left\{t_{n}\right\}$ be sequences of real numbers such that $s_{n} \geq 0$ for every $n \in \mathbb{N}$. Let $\left\{\beta_{n}\right\}$ be a sequence in $] 0,1[$ such that $\sum_{n=0}^{\infty} \beta_{n}=\infty$. Suppose that $s_{n+1} \leq\left(1-\beta_{n}\right) s_{n}+\beta_{n} t_{n}$ for every $n \in \mathbb{N}$. If $\lim \sup _{k \rightarrow \infty} t_{n_{k}} \leq 0$ for every nondecreasing sequence $\left\{n_{k}\right\}$ of $\mathbb{N}$ satisfying $\liminf _{k \rightarrow \infty}\left(s_{n_{k}+1}-s_{n_{k}}\right) \geq 0$, then $\lim _{n \rightarrow \infty} s_{n}=0$.

\section{Lemmas for a Finite Number of Resolvent Operators}

In this section, we prove some lemmas by using a finite number of resolvent operators for iterative schemes. Throughout this section, let $X$ be a CAT(1) space such that $d\left(v, v^{\prime}\right)<\pi / 2$ for every $v, v^{\prime} \in X$.

Lemma 7. For a given real number $\left.a \in] 0, \frac{1}{2}\right]$, let $\sigma \in[a, 1-a]$. For given points $y, y^{0}, y^{1} \in X$, define $w \in X$ by

$$
w=\sigma y^{0} \oplus(1-\sigma) y^{1} .
$$

Then,

$$
\cos d(w, y) \cos \left(a d\left(y^{0}, y^{1}\right)\right) \geq \min \left\{\cos d\left(y^{0}, y\right), \cos d\left(y^{1}, y\right)\right\}
$$

Proof. If $y^{0}=y^{1}$, it is obvious. Otherwise, by Lemma 1 , we have

$$
\begin{aligned}
\cos d & (w, y) \sin d\left(y^{0}, y^{1}\right) \\
& \geq \cos d\left(y^{0}, y\right) \sin \left(\sigma d\left(y^{0}, y^{1}\right)\right)+\cos d\left(y^{1}, y\right) \sin \left((1-\sigma) d\left(y^{0}, y^{1}\right)\right) \\
& \geq \min \left\{\cos d\left(y^{0}, y\right), \cos d\left(y^{1}, y\right)\right\}\left(\sin \left(\sigma d\left(y^{0}, y^{1}\right)\right)+\sin \left((1-\sigma) d\left(y^{0}, y^{1}\right)\right)\right) \\
& =2 \min \left\{\cos d\left(y^{0}, y\right), \cos d\left(y^{1}, y\right)\right\} \sin \frac{d\left(y^{0}, y^{1}\right)}{2} \cos \frac{(2 \sigma-1) d\left(y^{0}, y^{1}\right)}{2} .
\end{aligned}
$$

Dividing above by $2 \sin \left(d\left(y^{0}, y^{1}\right) / 2\right)$, we have

$$
\begin{aligned}
\cos d(w, y) \cos & \frac{d\left(y^{0}, y^{1}\right)}{2} \\
& \geq \min \left\{\cos d\left(y^{0}, y\right), \cos d\left(y^{1}, y\right)\right\} \cos \frac{(2 \sigma-1) d\left(y^{0}, y^{1}\right)}{2} \\
& \geq \min \left\{\cos d\left(y^{0}, y\right), \cos d\left(y^{1}, y\right)\right\} \cos \frac{(1-2 a) d\left(y^{0}, y^{1}\right)}{2} .
\end{aligned}
$$

Moreover, dividing above by $\cos \left((1-2 a) d\left(y^{0}, y^{1}\right) / 2\right)$, we have

$$
\begin{aligned}
& \min \left\{\cos d\left(y^{0}, y\right), \cos d\left(y^{1}, y\right)\right\} \\
& \leq \cos d(w, y) \frac{\cos \frac{(1-2 a) d\left(y^{0}, y^{1}\right)}{2} \cos \left(a d\left(y^{0}, y^{1}\right)\right)-\sin \frac{(1-2 a) d\left(y^{0}, y^{1}\right)}{2} \sin \left(a d\left(y^{0}, y^{1}\right)\right)}{\cos \frac{(1-2 a) d\left(y^{0}, y^{1}\right)}{2}} \\
& \leq \cos d(w, y) \cos \left(a d\left(y^{0}, y^{1}\right)\right) .
\end{aligned}
$$

This completes the proof.

Lemma 8. For a given real number $\left.a \in] 0, \frac{1}{2}\right]$, let $\sigma^{l} \in[a, 1-a]$ for every $l=0,1, \ldots, N-1$. For given points $y, y^{k} \in X$ for every $k=0,1, \ldots, N$, define $w^{l} \in X$ by

$$
w^{N}=y^{N} \text { and } w^{l}=\sigma^{l} y^{l} \oplus\left(1-\sigma^{l}\right) w^{l+1}
$$

for every $l=0,1, \ldots, N-1$. Then,

$$
\cos d\left(w^{0}, y\right) \cos \left(a d\left(y^{0}, w^{1}\right)\right) \geq \min _{k \in\{0,1, \ldots, N\}} \cos d\left(y^{k}, y\right) .
$$


Proof. By Lemma 7,

$$
\cos d\left(w^{0}, y\right) \cos \left(a d\left(y^{0}, w^{1}\right)\right) \geq \min \left\{\cos d\left(y^{0}, y\right), \cos d\left(w^{1}, y\right)\right\} .
$$

We also have

$$
\begin{aligned}
\cos d\left(w^{l}, y\right) & \geq \cos d\left(w^{l}, y\right) \cos \left(a d\left(y^{l}, w^{l+1}\right)\right) \\
& \geq \min \left\{\cos d\left(y^{l}, y\right), \cos d\left(w^{l+1}, y\right)\right\}
\end{aligned}
$$

for $l=1,2, \ldots, N-1$. Therefore, $\cos d\left(w^{0}, y\right) \cos \left(a d\left(y^{0}, w^{1}\right)\right) \geq \min _{k \in\{0,1, \ldots, N\}} \cos d\left(y^{k}, y\right)$. This completes the proof.

Corollary 1. Let $T^{k}$ be a quasi-nonexpansive mapping from $X$ to $X$ for every $k=0,1, \ldots, N$. For a given real number $\left.a \in] 0, \frac{1}{2}\right]$, let $\sigma^{l} \in[a, 1-a]$ for every $l=0,1, \ldots, N-1$. Define $U^{l}: X \rightarrow X$ by

$$
U^{N}=T^{N} \text { and } U^{l}=\sigma^{l} T^{l} \oplus\left(1-\sigma^{l}\right) U^{l+1}
$$

for every $l=0,1, \ldots, N-1$. Let $x \in X$ and $p \in \bigcap_{k=0}^{N} F\left(T^{k}\right)$. Then,

$$
\cos d\left(U^{0} x, p\right) \cos \left(a d\left(T^{0} x, U^{1} x\right)\right) \geq \cos d(x, p) .
$$

Next, we show several properties of a sequence of resolvents. Let $f$ be a proper lower semicontinuous convex function from $X$ into $]-\infty,+\infty]$ such that $\operatorname{argmin}_{X} f \neq \varnothing$ and let $\left\{\lambda_{n}\right\}$ be a real sequence such that $\inf \lambda_{n}>0$. Then we know that $\left\{R_{\lambda_{n} f}\right\}$ is a strongly quasi-nonexpansive sequence and $\Delta$-demiclosed sequence (see [11]). Therefore, we obtain the following results, using Lemma 4.

Lemma 9. Let $f^{k}$ be a proper lower semicontinuous convex function from $X$ into $\left.]-\infty,+\infty\right]$ for every $k=0,1, \ldots, N$ such that $\bigcap_{k=0}^{N} \operatorname{argmin}_{X} f^{k} \neq \varnothing$. For a given real number $\left.\left.a \in\right] 0, \frac{1}{2}\right]$, let $\sigma^{l} \in[a, 1-a]$ for every $l=0,1, \ldots, N-1$ and $\lambda^{k} \in[a,+\infty[$ for every $k=0,1, \ldots, N$. Let $R_{\lambda^{k} f^{k}}$ be the resolvent of $\lambda^{k} f^{k}$ for every $k=0,1, \ldots, N$. Define $U^{l}: X \rightarrow X$ by

$$
U^{N}=R_{\lambda^{N} f^{N}} \text { and } U^{l}=\sigma^{l} R_{\lambda^{l} f^{l}} \oplus\left(1-\sigma^{l}\right) U^{l+1}
$$

for every $l=0,1, \ldots, N-1$. Then

$$
F\left(U^{0}\right)=\bigcap_{k=0}^{N} \underset{X}{\operatorname{argmin}} f^{k} .
$$

Lemma 10. Let $\left\{T_{n}\right\}$ be a strongly quasi-nonexpansive sequence. Let $f$ be a proper lower semicontinuous convex function from $X$ into $]-\infty,+\infty]$ such that $\bigcap_{n=1}^{\infty} F\left(T_{n}\right) \cap \operatorname{argmin}_{X} f \neq \varnothing$. For a given real number $\left.a \in] 0, \frac{1}{2}\right]$, let $\left\{\sigma_{n}\right\} \subset[a, 1-a]$ and $\left\{\lambda_{n}\right\} \subset\left[a,+\infty\left[\right.\right.$. Let $R_{\lambda_{n} f}$ be the resolvent of $\lambda_{n} f$ for every $n \in \mathbb{N}$. Then $\left\{\sigma_{n} R_{\lambda_{n} f} \oplus\left(1-\sigma_{n}\right) T_{n}\right\}$ is a strongly quasi-nonexpansive sequence.

Proof. Let $V_{n}=\sigma_{n} R_{\lambda_{n} f} \oplus\left(1-\sigma_{n}\right) T_{n}$ for every $n \in \mathbb{N}$. From Lemma $4, V_{n}$ is a quasinonexpansive mapping for every $n \in \mathbb{N}$. From Corollary 1 , for $\left\{x_{n}\right\} \subset X$ and $p \in$ $\bigcap_{n=1}^{\infty} F\left(T_{n}\right) \cap \operatorname{argmin}_{X} f$ such that $\lim _{n \rightarrow \infty} \cos d\left(x_{n}, p\right) / \cos d\left(V_{n} x_{n}, p\right)=1$ and $\sup _{n \in \mathbb{N}} d\left(x_{n}, p\right)<$ $\pi / 2$, we have

$$
\cos d\left(V_{n} x_{n}, p\right) \cos \left(a d\left(R_{\lambda_{n} f} x_{n}, T_{n} x_{n}\right)\right) \geq \cos d\left(x_{n}, p\right)
$$

and thus

$$
\cos \left(a d\left(R_{\lambda_{n} f} x_{n}, T_{n} x_{n}\right)\right) \geq \frac{\cos d\left(x_{n}, p\right)}{\cos d\left(V_{n} x_{n}, p\right)} \rightarrow 1
$$


That is, $\lim _{n \rightarrow \infty} d\left(R_{\lambda_{n} f} x_{n}, T_{n} x_{n}\right)=0$. Therefore, we have

$$
\lim _{n \rightarrow \infty} d\left(T_{n} x_{n}, V_{n} x_{n}\right)=\lim _{n \rightarrow \infty} \sigma_{n} d\left(R_{\lambda_{n} f} x_{n}, T_{n} x_{n}\right)=0 .
$$

As $1=\lim _{n \rightarrow \infty} \cos d\left(x_{n}, p\right) / \cos d\left(V_{n} x_{n}, p\right)=\lim _{n \rightarrow \infty} \cos d\left(x_{n}, p\right) / \cos d\left(T_{n} x_{n}, p\right)$, we have

$$
\lim _{n \rightarrow \infty} d\left(T_{n} x_{n}, x_{n}\right)=0 .
$$

Thus, we obtain

$$
d\left(V_{n} x_{n}, x_{n}\right) \leq d\left(V_{n} x_{n}, T_{n} x_{n}\right)+d\left(T_{n} x_{n}, x_{n}\right) \rightarrow 0 .
$$

This completes the proof.

Corollary 2. Let $f^{k}$ be the same as in Lemma 9 for $k=0,1, \ldots, N$. For a given real number $\left.a \in] 0, \frac{1}{2}\right]$, let $\left\{\sigma_{n}^{l}\right\} \subset[a, 1-a]$ for every $l=0,1, \ldots, N-1$ and $\left\{\lambda_{n}^{k}\right\} \subset[a,+\infty[$ for every $k=0,1, \ldots, N$. Let $R_{\lambda_{n}^{k} f^{k}}$ be the resolvent of $\lambda_{n}^{k} f^{k}$ for every $k=0,1, \ldots, N$ and $n \in \mathbb{N}$. Define $U_{n}^{l}: X \rightarrow X$ by

$$
U_{n}^{N}=R_{\lambda_{n}^{N} f^{N}} \text { and } U_{n}^{l}=\sigma_{n}^{l} R_{\lambda_{n}^{l} f^{l}} \oplus\left(1-\sigma_{n}^{l}\right) U_{n}^{l+1}
$$

for every $l=0,1, \ldots, N-1$ and $n \in \mathbb{N}$. Then, $\left\{U_{n}^{0}\right\}$ is a strongly quasi-nonexpansive sequence.

Lemma 11. Let $\left\{T_{n}\right\}$ be a quasi-nonexpansive and $\Delta$-demiclosed sequence. Let $f$ be a proper lower semicontinuous convex function from $X$ into $]-\infty,+\infty]$ such that $\bigcap_{n=1}^{\infty} F\left(T_{n}\right) \cap \operatorname{argmin}_{X} f \neq \varnothing$. For a given real number $\left.a \in] 0, \frac{1}{2}\right]$, let $\left\{\sigma_{n}\right\} \subset[a, 1-a]$ and $\left\{\lambda_{n}\right\} \subset\left[a,+\infty\left[\right.\right.$. Let $R_{\lambda_{n} f}$ be the resolvent of $\lambda_{n}$ f for every $n \in \mathbb{N}$. Then $\left\{\sigma_{n} R_{\lambda_{n} f} \oplus\left(1-\sigma_{n}\right) T_{n}\right\}$ is a $\Delta$-demiclosed sequence.

Proof. Let $V_{n}=\sigma_{n} R_{\lambda_{n} f} \oplus\left(1-\sigma_{n}\right) T_{n}$ for every $n \in \mathbb{N}$. Let $p \in \bigcap_{n=1}^{\infty} F\left(T_{n}\right) \cap \operatorname{argmin}_{X} f$, $\left\{x_{n}\right\} \subset X$, and $z \in X$ such that $\lim _{n \rightarrow \infty} d\left(V_{n} x_{n}, x_{n}\right)=0$ and suppose that $\left\{x_{n}\right\}$ is $\Delta$ convergent to $z$. Then,

$$
\cos d\left(V_{n} x_{n}, p\right) \cos \left(a d\left(R_{\lambda_{n} f} x_{n}, T_{n} x_{n}\right)\right) \geq \cos d\left(x_{n}, p\right)
$$

and thus

$$
\begin{aligned}
1 \geq \cos \left(a d\left(R_{\lambda_{n} f} x_{n}, T_{n} x_{n}\right)\right) & \geq \frac{\cos d\left(x_{n}, p\right)}{\cos d\left(V_{n} x_{n}, p\right)} \\
& \geq \frac{\cos \left(d\left(x_{n}, V_{n} x_{n}\right)+d\left(V_{n} x_{n}, p\right)\right)}{\cos d\left(V_{n} x_{n}, p\right)} \rightarrow 1 .
\end{aligned}
$$

Therefore, $\lim _{n \rightarrow \infty} d\left(R_{\lambda_{n} f} x_{n}, T_{n} x_{n}\right)=0$. Thus, we have

$$
\begin{aligned}
d\left(R_{\lambda_{n} f} x_{n}, V_{n} x_{n}\right) & =\left(1-\sigma_{n}\right) d\left(R_{\lambda_{n} f} x_{n}, T_{n} x_{n}\right) \\
& \leq(1-a) d\left(R_{\lambda_{n} f} x_{n}, T_{n} x_{n}\right) \rightarrow 0 .
\end{aligned}
$$

Since $R_{\lambda_{n} f}$ is a $\Delta$-demiclosed sequence, we have $R_{\lambda_{n} f} z=z$. Similarly,

$$
\begin{aligned}
d\left(T_{n} x_{n}, V_{n} x_{n}\right) & =\sigma_{n} d\left(R_{\lambda_{n} f} x_{n}, T_{n} x_{n}\right) \\
& \leq(1-a) d\left(R_{\lambda_{n} f} x_{n}, T_{n} x_{n}\right) \rightarrow 0 .
\end{aligned}
$$

Since $\left\{T_{n}\right\}$ is a $\Delta$-demiclosed sequence, we have $T_{n} z=z$. Thus, $V_{n} z=z$. This completes the proof.

Corollary 3. Let $f^{k},\left\{\sigma_{n}^{l}\right\},\left\{\lambda_{n}^{k}\right\}$ and $\left\{U_{n}^{l}\right\}$ be the same as in Corollary 2 for $k=0,1, \ldots, N$ and $l=0,1, \ldots, N-1$. Then $\left\{U_{n}^{0}\right\}$ is a $\Delta$-demiclosed sequence. 


\section{Iterative Schemes for a Finite Resolvents Operators}

We prove convergence of Mann and Halpern types of iterative sequences for finitely many convex functions by using the properties of a sequence of the resolvents in CAT(1) space.

Theorem 1. Let $X$ be a complete CAT(1) space such that $d\left(v, v^{\prime}\right)<\pi / 2$ for every $v, v^{\prime} \in X$. Let $f^{k}$ be a proper lower semicontinuous convex function from $X$ into $\left.]-\infty,+\infty\right]$ for every $k=$ $0,1, \ldots, N$ such that $F=\bigcap_{k=0}^{N} \operatorname{argmin}_{X} f^{k} \neq \varnothing$. For a given real number $\left.\left.a \in\right] 0, \frac{1}{2}\right]$, let $\left\{\sigma_{n}^{l}\right\} \subset[a, 1-a]$ for every $l=0,1, \ldots, N-1$ and $\left\{\lambda_{n}^{k}\right\} \subset[a,+\infty[$ for every $k=0,1, \ldots, N$. Let $R_{\lambda_{n}^{k} f^{k}}$ be the resolvent of $\lambda_{n}^{k} f^{k}$ for every $k=0,1, \ldots, N$ and $n \in \mathbb{N}$. Define $U_{n}^{l}: X \rightarrow X$ by

$$
U_{n}^{N}=R_{\lambda_{n}^{N} f^{N}} \text { and } U_{n}^{l}=\sigma_{n}^{l} R_{\lambda_{n}^{l} f^{l}} \oplus\left(1-\sigma_{n}^{l}\right) U_{n}^{l+1}
$$

for every $l=0,1, \ldots, N-1$ and $n \in \mathbb{N}$. Let $\left\{\alpha_{n}\right\}$ be a real sequence in $[a, 1-a]$. For a given point $x_{1} \in X$, let $\left\{x_{n}\right\}$ be the sequence in $X$ generated by

$$
x_{n+1}=\alpha_{n} x_{n} \oplus\left(1-\alpha_{n}\right) U_{n}^{0} x_{n}
$$

for $n \in \mathbb{N}$. Then, $\left\{x_{n}\right\} \Delta$-converges to a point of $F$.

Proof. Let $z \in F$. As $U_{n}^{0}$ is a quasi-nonexpansive mapping, it follows from Lemma 2 that

$$
\begin{aligned}
\cos d\left(x_{n+1}, z\right) & \geq \alpha_{n} \cos d\left(x_{n}, z\right)+\left(1-\alpha_{n}\right) \cos d\left(U_{n}^{0} x_{n}, z\right) \\
& \geq \cos d\left(x_{n}, z\right) .
\end{aligned}
$$

Thus we have $d\left(x_{n+1}, z\right) \leq d\left(x_{n}, z\right)$ for $n \in \mathbb{N}$. There exists $D=\lim _{n \rightarrow \infty} d\left(x_{n}, z\right) \leq$ $d\left(x_{1}, z\right)<\pi / 2$. From Lemma 1 , we get

$$
\begin{aligned}
& \cos d\left(x_{n+1}, z\right) \sin d\left(x_{n}, U_{n}^{0} x_{n}\right) \\
& \quad \geq \cos d\left(x_{n}, z\right) \sin \alpha_{n} d\left(x_{n}, U_{n}^{0} x_{n}\right)+\cos d\left(U_{n}^{0} x_{n}, z\right) \sin \left(1-\alpha_{n}\right) d\left(x_{n}, U_{n}^{0} x_{n}\right) \\
& \quad \geq 2 \cos d\left(x_{n}, z\right) \sin \frac{d\left(x_{n}, U_{n}^{0} x_{n}\right)}{2} \cos \frac{\left(2 \alpha_{n}-1\right) d\left(x_{n}, U_{n}^{0} x_{n}\right)}{2} .
\end{aligned}
$$

If $d\left(x_{n}, U_{n}^{0} x_{n}\right) \neq 0$, we obtain

$$
\cos d\left(x_{n+1}, z\right) \cos \frac{d\left(x_{n}, U_{n}^{0} x_{n}\right)}{2} \geq \cos d\left(x_{n}, z\right) \cos \frac{\left(2 \alpha_{n}-1\right) d\left(x_{n}, U_{n}^{0} x_{n}\right)}{2} .
$$

As $\left\{\alpha_{n}\right\} \subset[a, 1-a]$, we get

$$
1>\frac{\cos \frac{d\left(x_{n}, U_{n}^{0} x_{n}\right)}{2}}{\cos \frac{(1-2 a) d\left(x_{n}, U_{n}^{0} x_{n}\right)}{2}} \geq \frac{\cos \frac{d\left(x_{n}, U_{n}^{0} x_{n}\right)}{2}}{\cos \frac{\left(2 \alpha_{n}-1\right) d\left(x_{n}, U_{n}^{0} x_{n}\right)}{2}} \geq \frac{\cos d\left(x_{n}, z\right)}{\cos d\left(x_{n+1}, z\right)} .
$$

As $D=\lim _{n \rightarrow \infty} d\left(x_{n}, z\right) \leq d\left(x_{1}, z\right)<\pi / 2$, we have

$$
\lim _{n \rightarrow \infty} \frac{\cos \frac{d\left(x_{n}, U_{n}^{0} x_{n}\right)}{2}}{\cos \frac{(1-2 a) d\left(x_{n}, U_{n}^{0} x_{n}\right)}{2}}=1
$$

and thus $\lim _{n \rightarrow \infty} d\left(x_{n}, U_{n}^{0} x_{n}\right)=0$. Let $x_{0}$ be an asymptotic center of $\left\{x_{n}\right\}$ and $y$ an asymptotic center of any subsequence $\left\{x_{n_{k}}\right\} \subset\left\{x_{n}\right\}$. There exists $\left\{x_{n_{k_{l}}}\right\} \subset\left\{x_{n_{k}}\right\}$ such that $\left\{x_{n_{k_{l}}}\right\}$ 
$\Delta$-converges to $w$. As $\left\{U_{n_{k_{l}}}^{0}\right\}$ is a $\Delta$-demiclosed sequence and $\lim _{n \rightarrow \infty} d\left(U_{n_{k_{l}}}^{0} x_{n_{k_{l}}}, x_{n_{k_{l}}}\right)=0$, we obtain $w \in F$. Since there exists $\lim _{n \rightarrow \infty} d\left(x_{n_{k}}, w\right)$, we have

$$
\begin{aligned}
\limsup _{k \rightarrow \infty} d\left(x_{n_{k}}, w\right)=\lim _{k \rightarrow \infty} d\left(x_{n_{k}}, w\right) & =\lim _{l \rightarrow \infty} d\left(x_{n_{k_{l}}}, w\right) \\
& \leq \limsup _{l \rightarrow \infty} d\left(x_{n_{k_{l}}}, y\right) \leq \limsup _{k \rightarrow \infty} d\left(x_{n_{k}}, y\right) .
\end{aligned}
$$

Therefore, we obtain $y=w \in F$. Similarly, we get $x_{0}=y$. Therefore, $\left\{x_{n}\right\} \Delta$-converges to $x_{0} \in F$.

Theorem 2. Let $X, f^{k},\left\{\sigma_{n}^{l}\right\},\left\{\lambda_{n}^{k}\right\}$ and $\left\{U_{n}^{l}\right\}$ be the same as in Theorem 1 for $k=0,1, \ldots, N$ and $l=0,1, \ldots, N-1$. Let $\left\{\alpha_{n}\right\}$ be a real sequence in $] 0,1\left[\right.$ such that $\lim _{n \rightarrow \infty} \alpha_{n}=0$ and $\sum_{n=0}^{\infty} \alpha_{n}=\infty$. For given points $u, x_{1} \in X$, let $\left\{x_{n}\right\}$ be the sequence in $X$ generated by

$$
x_{n+1}=\alpha_{n} u \oplus\left(1-\alpha_{n}\right) U_{n}^{0} x_{n}
$$

for $n \in \mathbb{N}$. Suppose that one of the following conditions holds:

(a) $\sup _{v, v^{\prime} \in X} d\left(v, v^{\prime}\right)<\pi / 2$;

(b) $d\left(u, P_{F} u\right)<\pi / 4$ and $d\left(u, P_{F} u\right)+d\left(x_{0}, P_{F} u\right)<\pi / 2$;

(c) $\quad \sum_{n=0}^{\infty} \alpha_{n}^{2}=\infty$.

Then, $\left\{x_{n}\right\}$ converges to $P_{F} u$.

To prove this theorem, we also employ the technique proposed in [9]. Note that $F=\bigcap_{k=0}^{N} \operatorname{argmin}_{X} f^{k}$.

Proof. Let $p=P_{F} u$ and let

$$
\begin{aligned}
& s_{n}=1-\cos d\left(x_{n}, p\right), \\
& t_{n}=1-\frac{\cos d(u, p)}{\sin d\left(u, U_{n}^{0} x_{n}\right) \tan \left(\frac{\alpha_{n}}{2} d\left(u, U_{n}^{0} x_{n}\right)\right)+\cos d\left(u, U_{n}^{0} x_{n}\right)}, \\
& \beta_{n}= \begin{cases}1-\frac{\sin \left(\left(1-\alpha_{n}\right) d\left(u, U_{n}^{0} x_{n}\right)\right)}{\sin d\left(u, U_{n}^{0} x_{n}\right)} & \left(u \neq U_{n}^{0} x_{n}\right), \\
\alpha_{n} & \left(u=U_{n}^{0} x_{n}\right)\end{cases}
\end{aligned}
$$

for $n \in \mathbb{N}$. Since $U_{n}^{0}$ is a quasi-nonexpansive mapping, it follows from Lemma 3 that

$$
s_{n+1} \leq\left(1-\beta_{n}\right)\left(1-\cos d\left(U_{n}^{0} x_{n}, p\right)\right)+\beta_{n} t_{n} \leq\left(1-\beta_{n}\right) s_{n}+\beta_{n} t_{n}
$$

for $n \in \mathbb{N}$. By Lemma 2, we have

$$
\begin{aligned}
\cos d\left(x_{n+1}, p\right) & =\cos d\left(\alpha_{n} u \oplus\left(1-\alpha_{n}\right) U_{n}^{0} x_{n}, p\right) \\
& \geq \alpha_{n} \cos d(u, p)+\left(1-\alpha_{n}\right) \cos d\left(U_{n}^{0} x_{n}, p\right) \\
& \geq \alpha_{n} \cos d(u, p)+\left(1-\alpha_{n}\right) \cos d\left(x_{n}, p\right) \\
& \geq \min \left\{\cos d(u, p), \cos d\left(x_{n}, p\right)\right\}
\end{aligned}
$$

for $n \in \mathbb{N}$. So we have

$$
\cos d\left(x_{n}, p\right) \geq \min \left\{\cos d(u, p), \cos d\left(x_{0}, p\right)\right\}=\cos \max \left\{d(u, p), d\left(x_{0}, p\right)\right\}>0
$$

for $n \in \mathbb{N}$. Hence $\sup _{n \in \mathbb{N}} d\left(x_{n}, p\right) \leq \max \left\{d(u, p), d\left(x_{0}, p\right)\right\}<\pi / 2$. Next, we will show for each of the conditions (a-c) imply that $\sum_{n=0}^{\infty} \beta_{n}=\infty$. For the conditions (a) and (b), let 
$M=\sup _{n \in \mathbb{N}} d\left(u, U_{n}^{0} x_{n}\right)$. Thus, we will show $M<\pi / 2$. In case (a), it is obvious. In case (b), as $\sup _{n \in \mathbb{N}} d\left(x_{n}, p\right) \leq \max \left\{d(u, p), d\left(x_{0}, p\right)\right\}$, we have

$$
\begin{aligned}
M & \leq \sup _{n \in \mathbb{N}}\left(d(u, p)+d\left(U_{n}^{0} x_{n}, p\right)\right) \\
& \leq \sup _{n \in \mathbb{N}}\left(d(u, p)+d\left(x_{n}, p\right)\right) \\
& \leq \max \left\{2 d(u, p), d(u, p)+d\left(x_{0}, p\right)\right\}<\pi / 2 .
\end{aligned}
$$

Thus, for cases (a) and (b), we have

$$
\begin{aligned}
\beta_{n} & \geq 1-\frac{\sin \left(\left(1-\alpha_{n}\right) M\right)}{\sin M} \\
& =\frac{2}{\sin M} \sin \left(\frac{\alpha_{n}}{2} M\right) \cos \left(\left(1-\frac{\alpha_{n}}{2}\right) M\right) \\
& \geq \alpha_{n} \cos M
\end{aligned}
$$

for $n \in \mathbb{N}$. As $\sum_{n=0}^{\infty} \alpha_{n}=\infty$, each of the conditions (a) and (b) implies that $\sum_{n=0}^{\infty} \beta_{n}=\infty$. In the case (c), we have

$$
\beta_{n} \geq 1-\sin \frac{\left(1-\alpha_{n}\right) \pi}{2}=1-\cos \frac{\alpha_{n}}{2} \geq \frac{\alpha_{n}^{2} \pi^{2}}{16}
$$

for $n \in \mathbb{N}$. Hence the condition (c) also implies that $\sum_{n=0}^{\infty} \beta_{n}=\infty$. For $\left\{s_{n_{i}}\right\} \subset\left\{s_{n}\right\}$ with a nondecreasing real sequence $\left\{n_{i}\right\} \subset \mathbb{N}$ such that $\liminf _{i \rightarrow \infty}\left(s_{n_{i}+1}-s_{n_{i}}\right) \geq 0$, we have

$$
\begin{aligned}
0 & \leq \liminf _{i \rightarrow \infty}\left(s_{n_{i}+1}-s_{n_{i}}\right) \\
& =\liminf _{i \rightarrow \infty}\left(\cos d\left(x_{n_{i}}, p\right)-\cos d\left(x_{n_{i}+1}, p\right)\right) \\
& \leq \liminf _{i \rightarrow \infty}\left(\cos d\left(x_{n_{i}}, p\right)-\left(\alpha_{n_{i}} \cos d(u, p)+\left(1-\alpha_{n_{i}}\right) \cos d\left(U_{n_{i}}^{0} x_{n_{i}}, p\right)\right)\right) \\
& =\liminf _{i \rightarrow \infty}\left(\cos d\left(x_{n_{i}}, p\right)-\cos d\left(U_{n_{i}}^{0} x_{n_{i}}, p\right)\right) \\
& \leq \limsup _{i \rightarrow \infty}\left(\cos d\left(x_{n_{i}}, p\right)-\cos d\left(U_{n_{i}}^{0} x_{n_{i}}, p\right)\right) \leq 0
\end{aligned}
$$

Hence $\lim _{i \rightarrow \infty}\left(\cos d\left(x_{n_{i}}, p\right)-\cos d\left(U_{n_{i}}^{0} x_{n_{i}}, p\right)\right)=0$. Since $\sup _{n \in \mathbb{N}} d\left(U_{n}^{0} x_{n}, p\right)<\pi / 2$, we have $\lim _{i \rightarrow \infty}\left(\cos d\left(x_{n_{i}}, p\right) / \cos d\left(U_{n_{i}}^{0} x_{n_{i}}, p\right)\right)=1$. As $\left\{U_{n_{i}}^{0}\right\}$ is a strongly quasi-nonexpansive sequence, it follows that $\lim _{i \rightarrow \infty} d\left(x_{n_{i}}, U_{n_{i}}^{0} x_{n_{i}}\right)=0$. Let $\left\{x_{n_{j}}\right\} \subset\left\{x_{n_{i}}\right\}$ be a $\Delta$-convergent subsequence such that $\lim _{j \rightarrow \infty} d\left(u, x_{n_{j}}\right)=\liminf _{i \rightarrow \infty} d\left(u, x_{n_{i}}\right)$. Since $\left\{U_{n}^{0}\right\}$ is a $\Delta$-demiclosed sequence and $\lim _{j \rightarrow \infty} d\left(x_{n_{j}}, U_{n_{j}}^{0} x_{n_{j}}\right)=0$, the $\Delta$-limit $z \in\left\{x_{n_{j}}\right\}$ belongs to $F$. By Lemma 5 , we have

$$
\liminf _{i \rightarrow \infty} d\left(u, U_{n_{i}}^{0} x_{n_{i}}\right)=\liminf _{i \rightarrow \infty} d\left(u, x_{n_{i}}\right)=\lim _{j \rightarrow \infty} d\left(u, x_{n_{j}}\right) \geq d(u, z) \geq d(u, p) .
$$

Hence

$$
\begin{aligned}
\limsup _{i \rightarrow \infty} t_{n_{i}} & =\limsup _{i \rightarrow \infty}\left(1-\frac{\cos d(u, p)}{\sin d\left(u, U_{n_{i}}^{0} x_{n_{i}}\right) \tan \left(\frac{\alpha_{n_{i}}}{2} d\left(u, U_{n_{i}}^{0} x_{n_{i}}\right)+\cos d\left(u, U_{n_{i}}^{0} x_{n_{i}}\right)\right.}\right) \\
& =\limsup _{i \rightarrow \infty}\left(1-\frac{\cos d(u, p)}{\cos d\left(u, U_{n_{i}}^{0} x_{n_{i}}\right)}\right) \leq 0 .
\end{aligned}
$$

From Lemma 6, we have $\lim _{n \rightarrow \infty} s_{n}=0$. Therefore, $\left\{x_{n}\right\}$ converges to $p$. This completes the proof. 


\section{Applications to the Image Recovery Problem}

At the end of this work, we apply our results to the problem of finding a point of the intersection of a finite family of closed convex subsets. This problem is also known as the image recovery problem. See the works in $[21,22]$ and references therein.

Let $C$ be a nonempty closed convex subset of a complete CAT(1) space such that $d\left(v, v^{\prime}\right)<\pi / 2$ for every $v, v^{\prime} \in X$. Then, the indicator function $i_{C}: C \rightarrow X$ of $C$ defined by

$$
i_{C}(x)= \begin{cases}0 & (x \in C) \\ \infty & (x \notin C)\end{cases}
$$

is proper, lower semicontinuous, and convex. As is mentioned in [14], the resolvent $R_{i_{C}}$ of this function coincides with the metric projection $P_{C}$. Using this fact, we obtain the following results for the image recovery problem. The first result can be proved by using Theorem 1.

Theorem 3. Let $X$ be a complete CAT(1) space such that $d\left(v, v^{\prime}\right)<\pi / 2$ for every $v, v^{\prime} \in X$. Let $\left\{C_{0}, C_{1}, \ldots, C_{N}\right\}$ be a finite family of nonempty closed convex subsets of $X$ such that $C=$ $\bigcap_{k=0}^{N} C_{K} \neq \varnothing$. For a given real number $\left.\left.a \in\right] 0, \frac{1}{2}\right]$, let $\left\{\sigma_{n}^{l}\right\} \subset[a, 1-a]$ for $l=0,1, \ldots, N-1$ and $n \in \mathbb{N}$. Let $P_{C_{k}}$ be the metric projection onto $C_{k}$ for $k=0,1, \ldots, N$. Define $U_{n}^{l}: X \rightarrow X$ by

$$
U_{n}^{N}=P_{C_{N}} \text { and } U_{n}^{l}=\sigma_{n}^{l} P_{C_{l}} \oplus\left(1-\sigma_{n}^{l}\right) U_{n}^{l+1}
$$

for every $l=0,1, \ldots, N-1$ and $n \in \mathbb{N}$. Let $\left\{\alpha_{n}\right\}$ be a real sequence in $[a, 1-a]$. For a given point $x_{1} \in X$, let $\left\{x_{n}\right\}$ be the sequence in $X$ generated by

$$
x_{n+1}=\alpha_{n} x_{n} \oplus\left(1-\alpha_{n}\right) U_{n}^{0} x_{n}
$$

for $n \in \mathbb{N}$. Then, $\left\{x_{n}\right\} \Delta$-converges to a point of $C$.

Note that this theorem is a generalization of the result by [21] in the setting of Hilbert spaces, to complete CAT(1) spaces.

On the other hand, by using Thoerem 2, we can also prove the following theorem which was obtained by the authors of [23].

Theorem 4 (Kasahara-Kimura [23]). Let $X$ be a complete CAT(1) space such that $d\left(v, v^{\prime}\right)<$ $\pi / 2$ for every $v, v^{\prime} \in X$. Let $\left\{C_{0}, C_{1}, \ldots, C_{N}\right\}$ be a finite family of nonempty closed convex subsets of $X$ such that $C=\bigcap_{k=0}^{N} C_{K} \neq \varnothing$. For a given real number $\left.\left.a \in\right] 0, \frac{1}{2}\right]$, let $\left\{\sigma_{n}^{l}\right\} \subset[a, 1-a]$ for $l=0,1, \ldots, N-1$ and $n \in \mathbb{N}$. Let $P_{C_{k}}$ be the metric projection onto $C_{k}$ for $k=0,1, \ldots, N$. Define $U_{n}^{l}: X \rightarrow X$ by

$$
U_{n}^{N}=P_{C_{N}} \text { and } U_{n}^{l}=\sigma_{n}^{l} P_{C_{l}} \oplus\left(1-\sigma_{n}^{l}\right) U_{n}^{l+1}
$$

for every $l=0,1, \ldots, N-1$ and $n \in \mathbb{N}$. Let $\left\{\alpha_{n}\right\}$ be a real sequence in $] 0,1[$ such that $\lim _{n \rightarrow \infty} \alpha_{n}=0$ and $\sum_{n=0}^{\infty} \alpha_{n}=\infty$. For given points $u, x_{1} \in X$, let $\left\{x_{n}\right\}$ be the sequence in $X$ generated by

$$
x_{n+1}=\alpha_{n} u \oplus\left(1-\alpha_{n}\right) U_{n}^{0} x_{n}
$$

for $n \in \mathbb{N}$. Suppose that one of the following conditions holds:

(a) $\sup _{v, v^{\prime} \in X} d\left(v, v^{\prime}\right)<\pi / 2$;

(b) $d\left(u, P_{C} u\right)<\pi / 4$ and $d\left(u, P_{C} u\right)+d\left(x_{0}, P_{C} u\right)<\pi / 2$;

(c) $\sum_{n=0}^{\infty} \alpha_{n}^{2}=\infty$.

Then $\left\{x_{n}\right\}$ converges to $P_{C} u$. 


\section{Conclusions}

We proposed a new type of iterative scheme for the problem of finding a common minimizer of finitely many convex functions defined on a complete CAT(1) space. We considered the resolvent operators for proper lower semicontinuous convex functions defined on a complete CAT(1) space and their convex combination. As the convex combination on a CAT(1) space is defined only between two points, we need to take it repeatedly for three or more points.

In the first result (Theorem 1), we adopted a Mann-type sequence defined by the following iterative formula: $x_{1} \in X$ is given and

$$
x_{n+1}=\alpha_{n} x_{n} \oplus\left(1-\alpha_{n}\right) U_{n}^{0} x_{n}
$$

for $n \in \mathbb{N}$, where a mapping $U_{n}^{0}$ is defined by the convex combination of finitely many resolvents. Then, $\left\{x_{n}\right\}$ is $\Delta$-convergent to a solution to our problem.

In the second result (Theorem 2), we used a Halpern-type sequence defined as follows: $u, x_{1} \in X$ is given and

$$
x_{n+1}=\alpha_{n} u \oplus\left(1-\alpha_{n}\right) U_{n}^{0} x_{n}
$$

for $n \in \mathbb{N}$. Then, it converges to $P_{F} u$, the nearest point of the solution set $F$ to $u$.

Further, we showed that these results can be applied to the image recovery problem.

Author Contributions: The authors have contributed to this work on an equal basis. All authors read and approved the final manuscript.

Funding: This research received no external fundings.

Acknowledgments: The authors are grateful to anonymous referees for their valuable comments and suggestions.

Conflicts of Interest: The authors declare no conflict of interest.

\section{References}

1. Mann, W.R. Mean value methods in iteration; Proc. Am. Math. Soc. 1953, 4, 506-510. [CrossRef]

2. Halpern, B. Fixed points of nonexpanding maps. Bull. Am. Math. Soc. 1967, 73, 957-961. [CrossRef]

3. Reich, S. Weak convergence theorems for nonexpansive mappings in Banach spaces. J. Math. Anal. Appl. 1979, 67, 274-276. [CrossRef]

4. Takahashi; W.; Tamura, T. Convergence theorems for a pair of nonexpansive mappings. J. Convex Anal. 1998, 5, 45-56.

5. Wittmann, R. Approximation of fixed points of nonexpansive mappings. Arch. Math. 1992, 58, 486-491. [CrossRef]

6. Dhompongsa, S.; Panyanak, B. On $\Delta$-convergence theorems in CAT(0) spaces. Comput. Math. Appl. 2008, 56, 2572-2579. [CrossRef]

7. Saejung, S. Halpern's iteration in CAT(0) spaces. Fixed Point Theory Appl. 2010, 2010, 1471781. [CrossRef]

8. Piątek, B. Halpern iteration in CAT( $\kappa)$ spaces. Acta Math. Sin. Engl. Ser. 2011, 27, 635-646. [CrossRef]

9. Kimura, Y.; Satô, K. Halpern iteration for strongly quasinonexpansive mappings on a geodesic space with curvature bounded above by one. Fixed Point Theory Appl. 2013, 2013, 7. [CrossRef]

10. Kimura, Y.; Saejung, S.; Yotkaew, P. The Mann algorithm in a complete geodesic space with curvature bounded above. Fixed Point Theory Appl. 2013, 2013, 336. [CrossRef]

11. Kimura, Y.; Kohsaka, F. Two modified proximal point algorithms in geodesic spaces with curvature bounded above. Rend. Circ. Mat. Pallemo II Ser. 2019, 68, 83-104. [CrossRef]

12. Jost, J. Convex functionals and generalized harmonic maps into spaces of nonpositive curvature. Comment. Math. Helv. 1995, 70, 659-673. [CrossRef]

13. Mayer, U.F. Gradient flows on nonpositively curved metric spaces and harmonic maps. Comm. Anal. Geom. 1998, 6, 199-253. [CrossRef]

14. Kimura, Y.; Kohsaka, F. Spherical nonspreadingness of resolvents of convex functions in geodesic spaces. J. Fixed Point Theory Appl. 2016, 18, 93-115. [CrossRef]

15. Kimura Y.; Satô, K. Convergence of subsets of a complete geodesic space with curvature bounded above. Nonlinear Anal. 2012, 75, 5079-5085. [CrossRef]

16. Aoyama, K.; Kimura, Y. Strong convergence theorems for strongly nonexpansive sequences. Appl. Math. Comput. 2011, 217, 7537-7545. [CrossRef]

17. Kimura, Y.; Satô, K. Image recovery problem on a geodesic space with curvature bound above by one. In Proceedings of the Third Asian Conference on Nonlinear Analysis and Optimization, Matsue, Japan, 2-6 September 2012; pp. $165-172$. 
18. He, J.S.; Fang, D.H.; López, G.; Li, C. Mann's algorithm for nonexpansive mappings in CAT( $\kappa)$ spaces. Nonlinear Anal. 2012, 75, 445-452. [CrossRef]

19. Saejung, S.; Yotkaew, P. Approximation of zeros of inverse strongly monotone operators in Banach spaces. Nonlinear Anal. 2012, 75, 742-750. [CrossRef]

20. Aoyama, K.; Kimura, Y.; Kohsaka, F. Strong convergence theorems for strongly relatively nonexpansive sequences and applications. J. Nonlinear Anal. Optim. 2012, 3, 67-77.

21. Crombez, G. Image recovery by convex combinations of projections. J. Math. Anal. Appl. 1991, 155, 413-419. [CrossRef]

22. Kitahara, S.; Takahashi, W. Image recovery by convex combinations of sunny nonexpansive retractions. Topol. Methods Nonlinear Anal. 1993, 2, 333-342. [CrossRef]

23. Kasahara, K.; Kimura, Y. An iterative sequence for a finite number of metric projections on a complete geodesic space, Nolinear analysis and convex analysis. RIMS Kôkyûroku 2019, 2114, 120-126. 